\title{
Precision spectroscopy in light isovector, isoscalar and hybrid mesons
}

\author{
R. Morrin, Alan Ó Cais*ł M.B. Oktay, M.J. Peardon, S.M. Ryan, J-I. Skullerud \\ School of Mathematics, Trinity College, Dublin, Ireland \\ E-mail: alanc@maths.tcd.ie
}

\begin{abstract}
We discuss all-to-all propagators in the context of high statistical precision determinations of the isovector and isoscalar meson spectrum. In particular we look at the method of Ref. [2] that incorporates the low-lying eigenmodes and variance-reduced stochastic estimation. We find significant low-lying eigenmode dependence of mass determinations, with the number of required modes varying with the symmetry channel. We observe that the mass of a large number of traditionally noisy symmetry channels can be determined to 5\% statistical accuracy or higher in light-light mesons.
\end{abstract}

XXIVth International Symposium on Lattice Field Theory

July 23-28, 2006

Tucson, Arizona, USA

\footnotetext{
${ }^{*}$ Speaker.

${ }^{\dagger}$ Currently at CSSM, School of Chemstry and Physics, University of Adelaide, Adelaide SA5000, Australia
} 


\section{Introduction}

In meson spectroscopy, many symmetry channels can be easily explored by the use of extended operators that spatially separate the quark and antiquark. The possible gluonic paths connecting the pair give access to a new range of symmetry channels other than those achieved using only gamma matrix operations [1] and allows us to construct a large variational basis of operators. Unfortunately, the introduction of the gauge fields into the operator give rise to large fluctuations over an ensemble of gauge backgrounds. The use of all-to-all propagators is necessary to exploit translational invariance, utilise all information contained in the gauge fields and reduce the effect of these fluctuations in correlation functions.

As well as offering a method for high-accuracy measurements of isovector mesons, the practical all-to-all propagator scheme of Ref. [2] provides a framework to study isoscalar states in detail. The isoscalar mesons of QCD remain poorly understood phenomenologically. In particular, the $J^{P C}=0^{++}$sector contains a range of experimentally observed resonances that are difficult to interpret unambiguously in a quark model picture. These states have proved difficult to investigate in lattice simulations, since they require the calculation of disconnected correlation functions of quark propagators, that are inaccessible to the usual point propagator method of lattice QCD. The disconnected correlation functions, like those of the extended operators, suffer from having large fluctuations over an ensemble of gauge backgrounds.

Here, we present preliminary results from an investigation into the statistical accuracy achieveable using this method for a wide range of states in isovector channels. We evaluate the low-lying eigenmode dependence of correlation functions for some of these channels and also look at the $0^{++}$ and $\eta^{\prime}$ isoscalar channels.

\section{The all-to-all propagator}

In order to investigate correlation functions of all symmetry channels of isovector and isoscalar mesons, access to all entries of the quark propagator is desirable to exploit the self-averaging effects of translational invariance and allow the straight forward implementation of extended operators. Many algorithms to compute or estimate these have been devised, for example see Refs. [3, 4]. In this work, we utilise an exact implementation which employs a hybrid method that combines an eigenvector decomposition with a variance-reduced stochastic estimator [2].

To construct the all-to-all propagator, the lowest $N_{\text {ev }}$ eigenmodes of the hermitian Dirac matrix $Q=\gamma_{5} M$ are first computed, and a truncated spectral decomposition of the propagator is then given by

$$
\bar{Q}_{0}=\sum_{i}^{N_{\mathrm{ev}}} \frac{1}{\lambda_{i}} v^{(i)} \otimes v^{(i)^{\dagger}} .
$$

If $N_{\mathrm{ev}}$ is equal to the dimension of the matrix, then $\bar{Q}_{0}=Q^{-1}$, otherwise the propagator can be expressed as

$$
Q^{-1}=\bar{Q}_{0}+\bar{Q}_{1}
$$

and the truncation in the eigenvector representation can be corrected by estimating $\bar{Q}_{1}$ stochastically. To construct a stochastic estimate of the inverse of a matrix, $A$, an ensemble of random noise 
vectors, $\left\{\eta_{[1]}, \cdots, \eta_{\left[N_{R}\right]}\right\}$ with the property

$$
\left\langle\left\langle\eta(x) \otimes \eta(y)^{\dagger}\right\rangle\right\rangle=\delta_{x, y},
$$

where $\langle\langle\ldots\rangle\rangle$ denotes the ensemble average, is generated. The solution vectors, $\psi_{[R]}(x)=A^{-1} \eta_{[R]}(y)$, can then be computed, typically using a conjugate gradient algorithm, and $A^{-1}$ is estimated from

$$
A^{-1} \simeq \frac{1}{N_{R}} \sum_{R}^{N_{R}} \psi_{[R]} \otimes \eta_{[R]}^{\dagger}
$$

If we let $X(x, y)=\eta(x) \eta(y)^{\dagger}$, the variance of the trace of the matrix inverse due to the stochastic estimation is essentially composed of two summations [5],

$$
\begin{aligned}
\operatorname{Var}\left[\operatorname{Tr}\left(A^{-1} X\right)\right] \equiv & \left\langle\left\langle\left|\sum_{x, y} A^{-1}(x, y) X(y, x)-\operatorname{Tr}\left(A^{-1}\right)\right|^{2}\right\rangle\right\rangle \\
= & \left.\sum_{x \neq y}\left(\left\langle\left\langle|X(y, x)|^{2}\right\rangle\right\rangle\left|A^{-1}(x, y)\right|^{2}+A^{-1}(x, y) A^{-1}(y, x)\right)^{\dagger}\left\langle\left\langle(X(x, y))^{2}\right\rangle\right\rangle\right) \\
& +\sum_{x}\left\langle\left\langle\mid\left(X(x, x)-\left.1\right|^{2}\right\rangle\right\rangle\left|A^{-1}(x, x)\right|^{2} .\right.
\end{aligned}
$$

The action of the matrix to be estimated, $\bar{Q}_{1}$, can be implemented by noting that

$$
\bar{Q}_{1}=\mathscr{P}_{1} Q^{-1},
$$

where $\mathscr{P}_{1}$ is a projection operator onto the vector space orthogonal to the $N_{\mathrm{ev}}$ eigenvectors, so

$$
\mathscr{P}_{1}=1-\mathscr{P}_{0}=1-\sum_{i}^{N_{\mathrm{ev}}} v^{(i)} \otimes v^{(i)^{\dagger}}
$$

and the action of $\mathscr{P}_{1}$ is equivalent to Gram-Schmidt orthogonalisation with respect to the computed eigenvectors.

For accessible numbers of eigenvectors, the stochastic estimator in its simplest form is noisy. We choose to "dilute" the noise vector, which results in rapid variance reduction. In this context, dilution means creating a set of noise vectors by applying a set of masks to a single noise source. These masks might for example select a particular time-slice of the vector (referred to as time dilution), thus returning $N_{\mathrm{T}}$ noise vectors from each single noise source.

One can then decompose the ensemble of noise vectors into each of the $N_{\text {dil }}$ dilution subspaces, $\eta_{[i]}=\sum_{r=1}^{N_{\text {dil }}} \eta_{[i]}^{(r)}$, yielding an ensemble,

$$
\left\{\left\{\eta_{[1]}^{(1)}, \eta_{[1]}^{(2)}, \cdots, \eta_{[1]}^{\left(N_{\text {dii }}\right)}\right\},\left\{\eta_{[2]}^{(1)}, \eta_{[2]}^{(2)}, \cdots, \eta_{[2]}^{\left(N_{\text {dil }}\right)}\right\}, \cdots,\left\{\eta_{\left[N_{R}\right]}^{(1)}, \eta_{\left[N_{R}\right]}^{(2)}, \cdots, \eta_{\left[N_{R}\right]}^{\left(N_{\text {dil }}\right)}\right\}\right\} .
$$

Additional to Eqn. (2.3), the subspace dilution now implies

$$
\eta^{(r)}(x) \eta^{(t)}(y)^{\dagger}=X^{(r, t)}(x, y)=X^{(r, r)}(x, y) \delta^{(r, t)}
$$

even before expectation values are taken. In Eqn. (2.5), we can see that dilution will, therefore, reduce the first summation, $\sum_{x \neq y}$, to a sum where $x$ and $y$ are in the same dilution subspace before expectation values are taken. This reduction in the summation is true for any white noise and in the 
homeopathic limit of $N_{\mathrm{dil}}=N$ dilution subspaces this first sum is automatically zero since $x$ and $y$ can no longer be in the same dilution subspace. The second sum is proportional to the square of the diagonal error only, for $Z(N)$ noise this error is zero and we have zero variance in the homeopathic limit.

In principle, the method of dilution can be optimised to reduce the variance. Time dilution has been seen to be crucial and leads to an exponential error reduction. While the variance falls off more rapidly than $1 / \sqrt{N}$ for all further dilutions, little dependence on the details of this further dilution has been observed in simulations performed to date.

\section{An efficient implementation}

The method can be implemented efficiently in software by use of a "hybrid list" method. Two lists of $N_{\mathrm{HL}}=N_{\mathrm{ev}}+N_{\text {dil }}$ vectors $u$ and $w$ are written,

$$
\begin{aligned}
& w^{(i)}=\left\{\frac{v^{(1)}}{\lambda_{1}}, \cdots, \frac{v^{\left(N_{\mathrm{ev}}\right)}}{\lambda_{N_{\mathrm{ev}}}}, \eta^{(1)}, \cdots, \eta^{\left(N_{\mathrm{dil}}\right)}\right\} \\
& u^{(i)}=\left\{v^{(1)}, \cdots, v^{\left(N_{\mathrm{ev}}\right)}, \psi^{(1)}, \cdots, \psi^{\left(N_{\mathrm{dil}}\right)}\right\},
\end{aligned}
$$

Then the inverse of $M$ is written as a single sum over the pair of lists

$$
M^{-1}=\sum_{i=1}^{N_{\mathrm{HL}}}\left(u^{(i)} \otimes w^{(i) \dagger}\right) \gamma_{5} .
$$

To compute a two-point correlation function for an isovector meson requires two further steps. Pairs of hybrid lists from two independent noise sources, $r$ and $s$, are combined to form an $N_{\mathrm{HL}} \times$ $N_{\mathrm{HL}}$ matrix or operator field,

$$
\mathscr{O}_{[r, s]}^{(i, j) A}(t)=w_{[r]}^{(i) *}(t) \gamma_{5} \Gamma_{A} u_{[s]}^{(j)}(t)
$$

where $\Gamma_{A}$ is an operator on a time-slice that creates a meson with the desired quantum numbers. This stage of the construction is the only point where this operator is invoked, and so an efficient user-friendly implementation can be written. The end user need only write a function to perform the operation of Eqn. (3.4), and this is a simple task for most mesons.

The two-point correlation function between source $A$ and $\operatorname{sink} B$ is computed from

$$
C_{A B}(\Delta t)=\sum_{i, j, t} \mathscr{O}_{[r, s]}^{(i, j) A}(t) \mathscr{O}_{[s, r]}^{(j, i) B}(t+\Delta t) .
$$

Since most operators can be constructed to have positive hermiticity we can achieve a large variational basis simply by combining different source and sink operator fields as long as we ensure that the sample and hybrid list indices are summed correctly.

For isoscalar mesons, the disconnected diagram must also be computed. This can be done very straightforwardly using the hybrid list trick. The disconnected two-point function for source $A$ and $\operatorname{sink} B$ is

$$
C_{A B}(\Delta t)=\sum_{i, j, t} \mathscr{O}_{[r, r]}^{(i, i) A}(t) \mathscr{O}_{[s, s]}^{(j, j) B}(t+\Delta t) .
$$




\section{Results}

The method has been employed in a preliminary computation of the masses of isovector and isoscalar mesons of $N_{f}=2$ dynamical QCD on a tuned anisotropic lattice [7] with renormalised anisotropy, $\xi=6$ and a lattice spacing of $a_{s} \sim 0.17 \mathrm{fm}\left(a_{t}^{-1} \sim 7 \mathrm{GeV}\right)$. An ensemble of between $225-2508^{3} \times 80$ and $12^{3} \times 80$ lattices was used, with a quark mass around that of the strange. The details of the simulation parameters can be found in [6].

The spectrum of light and heavy-light mesons was computed using the method, including the S- and P-wave quark model states and an exotic hybrid, with quantum numbers $1^{-+}$.

\subsection{Eigenmode dependence}

It has been shown, in [2], that the explicit inclusion of low-lying eigenmodes can dramatically improve the determined statistical error of correlation functions. To investigate the number of eigenmodes necessary to achieve this improvement we look at the behaviour of the fitted mass in a constant fitting region while varying the number of eigenmodes (and also the dilution level) for various symmetry channels. The fitting region is chosen according to the best fit achievable with the highest dilution and number of eigenmodes. As an illustration of the observed behaviour we show two channels, the $1^{--}$and $2^{-+}$, in Fig. (1).
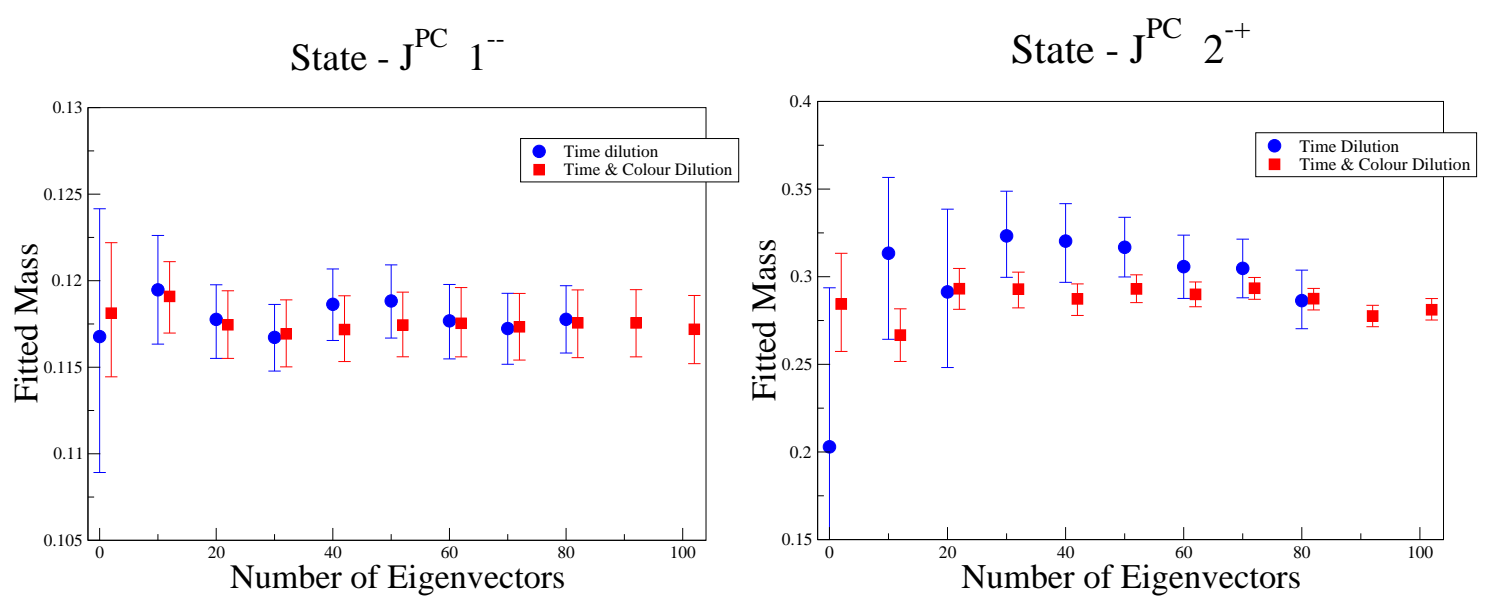

Figure 1: The variation, in a constant fitting region, of the fitted mass with the number of low-lying eigenmodes and two levels of dilution for the light-light $1^{--}$and $2^{-+}$channels.

We observe state-dependent behaviour in our determinations, with greater dilution and number of eigenmode dependence in the (extended-operator) $2^{-+}$channel. However, in both cases we observe a saturation of improvement from the eigenmodes far below the maximum. It would seem that as low as 20 eigenmodes with colour dilution might achieve optimal results. These calculations were done on the $8^{3} \times 80$ and there is a heuristic argument that the number of eigenmodes to achieve these levels of improvement should scale with the volume.

\subsection{Isovector correlators}

Isovector correlators are calculated using the variational approach with 4 different smearing operators for both light-light and heavy-light mesons on the two different volumes. The preliminary 
operators, correlated fitted masses and $\chi^{2}$ values can be found in Table 1 . The fit ranges and values are determined using sliding window fits as detailed in [6]. The percentage errors on the fitted mass are displayed in Fig. 2 for both volumes. A fuller analysis with a larger variational basis is currently underway.

\begin{tabular}{|l|l||l|l|l|l|l|l|l|l|l|}
\cline { 3 - 10 } \multicolumn{2}{c|}{} & \multicolumn{2}{l|}{ Heavy-light } & \multicolumn{2}{l|}{ Light-light } \\
\cline { 3 - 10 } \multicolumn{2}{l|}{} & \multicolumn{2}{l|}{ Volume $8^{3} \times 80$} & \multicolumn{2}{l|}{ Volume $12^{3} \times 80$} & \multicolumn{2}{|l|}{ Volume $8^{3} \times 80$} & \multicolumn{2}{l|}{ Volume $12^{3} \times 80$} \\
\hline State & Operator & $a_{t} m_{\text {fitted }}$ & $\chi_{\text {PDOF }}^{2}$ & $a_{t} m_{\text {fitted }}$ & $\chi_{\text {PDOF }}^{2}$ & $a_{t} m_{\text {fitted }}$ & $\chi_{\text {PDOF }}^{2}$ & $a_{t} m_{\text {fitted }}$ & $\chi_{\text {PDOF }}^{2}$ \\
\hline \hline $0^{-+}$ & $\gamma_{5}$ & $0.2396_{-7}^{+7}$ & 0.08 & $0.2393_{-4}^{+4}$ & 0.41 & $0.057_{-2}^{+1}$ & 0.24 & $0.0540_{-2}^{+2}$ & 0.01 \\
$1^{--}$ & $\vec{\gamma}$ & $0.2511_{-10}^{+10}$ & 0.28 & $0.2530_{-4}^{+4}$ & 0.46 & $0.105_{-3}^{+3}$ & 0.26 & $0.1058_{-7}^{+7}$ & 0.22 \\
$0^{++}$ & 1 & $0.2774_{-19}^{+20}$ & 0.01 & $0.315_{-13}^{+12}$ & 0.33 & $0.161_{-3}^{+3}$ & 0.65 & $0.098_{-5}^{+6}$ & 0.18 \\
$1^{+-}$ & $\gamma_{5} \vec{p}$ & $0.2988_{-30}^{+28}$ & 0.28 & $0.3246_{-13}^{+12}$ & 0.47 & $0.154_{-7}^{+7}$ & 0.61 & $0.176_{-6}^{+6}$ & 0.13 \\
$1^{++}$ & $\vec{\gamma} \times \vec{p}$ & $0.2773_{-41}^{+46}$ & 0.21 & $0.3104_{-20}^{+21}$ & 0.50 & $0.148_{-2}^{+2}$ & 0.28 & $0.138_{-5}^{+5}$ & 0.12 \\
$2^{++}$ & $\gamma_{z} p_{x}+\gamma_{x} p_{z}$ & $0.3242_{-14}^{+14}$ & 0.39 & $0.3285_{-75}^{+72}$ & 0.31 & $0.217_{-5}^{+4}$ & 0.15 & $0.216_{-2}^{+2}$ & 0.46 \\
$2^{--}$ & $\gamma_{5}\left(s_{x}-s_{y}\right)$ & $0.3810_{-38}^{+36}$ & 0.22 & $0.3914_{-98}^{+95}$ & 0.31 & $0.214_{-13}^{+14}$ & 0.42 & $0.267_{-9}^{+9}$ & 0.21 \\
$2^{-+}$ & $\gamma_{z}\left(s_{x}-s_{y}\right)$ & $0.3664_{-87}^{+87}$ & 0.42 & $0.391_{-13}^{+11}$ & 0.41 & $0.240_{-18}^{+18}$ & 0.16 & $0.273_{-4}^{+4}$ & 0.46 \\
$1^{-+}$ & $\vec{\gamma} \times \vec{u}$ & $0.324_{-30}^{+32}$ & 0.31 & $0.271_{-34}^{+30}$ & 0.23 & $0.218_{-25}^{+25}$ & 0.05 & $0.144_{-46}^{+40}$ & 0.29 \\
\hline
\end{tabular}

Table 1: Table of fitted masses for the states in Fig. 2. Also shown is the operator used to obtain them and the $\chi^{2}$ per degree of freedom. The extended operators $\vec{p}, \vec{s}$ and $\vec{u}$ are as defined in Ref. [1].
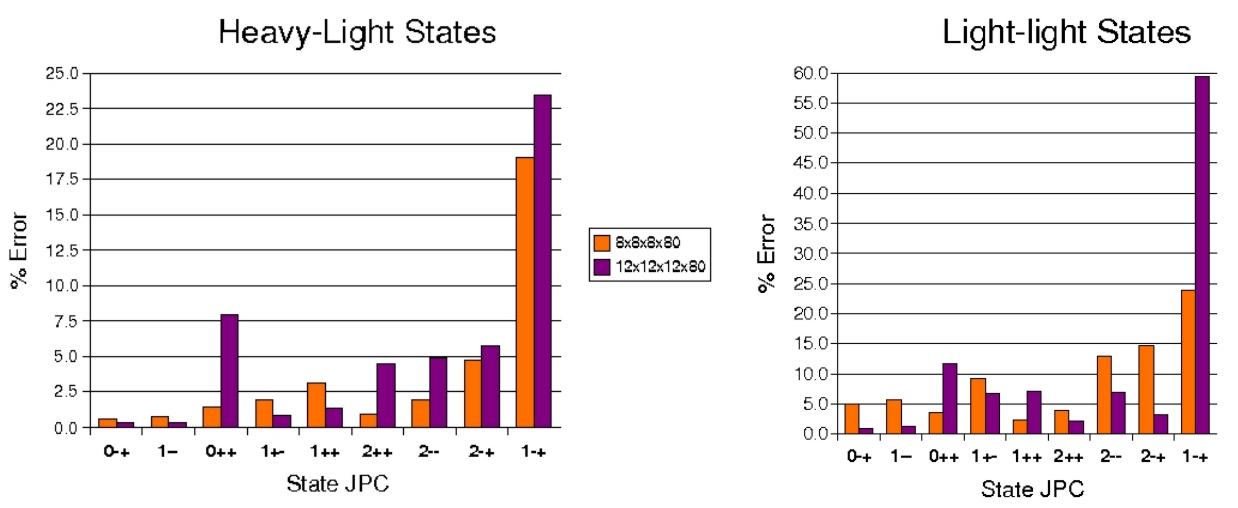

Figure 2: Percentage error of fitted masses of some symmetry channels using the all-to-all method for heavy-light and light-light states on two different lattice volumes

We can see that the statistical accuracy to which the fitted masses are determined is extremely high, $5 \%$ or lower for the vast majority of cases. Most notable however is the large error on the determined mass of the hybrid $1^{-+}$, it is far above the other states and the excited gluonic path appears to have a large effect on its determination.

\subsection{Isoscalar correlators}

In Fig. 3 we have the sliding window plots of the isoscalar $0^{-+}$(left) and $0^{++}$(right) mesons. Plotted are the masses determined using the 100 lowest-lying eigenvectors in the hybrid method but without a variational basis. These results are incomplete and preliminary and are currently being investigated further. Dilutions more suited to the nature of the disconnected diagrams need to be investigated. 

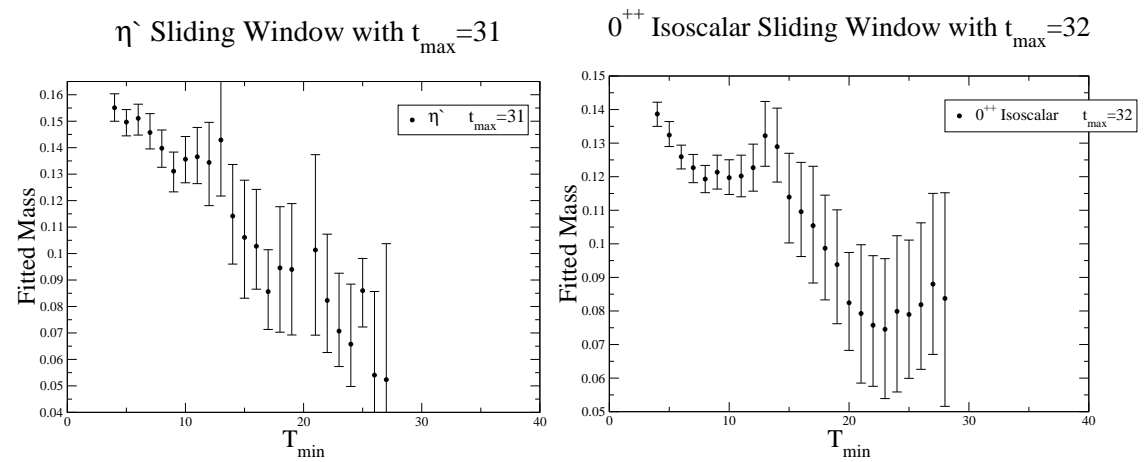

Figure 3: Masses of the isoscalar $0^{-+}$(left) and $0^{++}$mesons determined in a sliding window fit.

These measurements were taken on the $8^{3} \times 80$ lattices and, as a general comment, the $\eta^{\prime}$ is heavier than the pion and the $0^{++}$isoscalar appears lighter than its isovector partner.

\section{Conclusions}

All-to-all propagator methods make it possible to use all available information contained in background gauge configurations, which are an essential component of isoscalar channel studies. The hybrid method of Ref. [2] allows us to extract the important physics from the low-lying eigenmodes and combine this with a noisy correction step in a natural way. The framework is straightforwardly applicable to decays, glueball mixing, baryons and thermodynamic quantities (condensates and susceptibilities). Preliminary investigations with this method suggest it provides a toolkit to make accurate measurements of the properties of the isoscalar and isovector mesons of QCD even with a low number of low-lying eigenmodes.

\section{References}

[1] P. Lacock, C. Michael, P. Boyle and P. Rowland [UKQCD Collaboration], Phys. Rev. D 54 (1996) 6997 [arXiv:hep-lat/9605025].

[2] J. Foley, K. Jimmy Juge, A. Ó Cais, M. Peardon, S. M. Ryan and J. I. Skullerud, Comput. Phys. Commun. 172 (2005) 145 [arXiv:hep-lat/0505023].

[3] H. Neff, N. Eicker, T. Lippert, J. W. Negele and K. Schilling, Phys. Rev. D 64, 114509 (2001).

[4] W. Wilcox, arXiv:hep-lat/9911013.

[5] S. Bernardson, P. McCarty and C. Thron, Comput. Phys. Commun. 78 (1993) 256.

[6] M. B. Oktay et al., "The spectrum of radial, orbital and gluoinc excitations of charmonium.", to appear in the proceeding of Lattice 2006.

[7] R. Morrin, A. O. Cais, M. Peardon, S. M. Ryan and J. I. Skullerud, Phys. Rev. D 74 (2006) 014505 [arXiv:hep-lat/0604021]. 